\title{
STATUS AND CHALLENGES OF THE LHC CONSTRUCTION
}

\author{
R. Ostojic, CERN, Geneva, Switzerland
}

\begin{abstract}
The LHC is designed to provide proton beams of $7 \mathrm{TeV}$ and nominal luminosity of $10^{34} \mathrm{~cm}^{-2} \mathrm{~s}^{-1}$. This objective is achieved at an affordable cost by pushing all major collider components to the limits of technology, by upgrading the existing CERN accelerators and infrastructure, and by involving the technical expertise, resources and dedication of accelerator laboratories world-wide. Following a decade of intensive $R \& D$ and technical validation of major collider systems, the LHC construction is now fully underway. Major industrial contracts have been awarded and are in execution for the procurement of the magnet, cryogenics and other systems. In this report, the status of the design and construction of the major LHC systems is presented.
\end{abstract}

\section{INTRODUCTION}

The Large Hadron Collider, under construction in CERN, is designed to provide proton-proton collisions at centre-of-mass energy of $14 \mathrm{TeV}$ and luminosity of $10^{34} \mathrm{~cm}^{-2} \mathrm{~s}^{-1}$ [1]. The LHC consists of two interleaved synchrotron rings, whose main elements are the two-in-one superconducting dipoles and quadrupoles operating in superfluid helium at a temperature of $1.9 \mathrm{~K}$. The collider will be installed in the existing tunnel of $26.7 \mathrm{~km}$ circumference, which until recently housed the LEP collider. The layout of the machine closely mirrors that of LEP, with eight identical arcs, each $2.8 \mathrm{~km}$ long, separated by eight $540 \mathrm{~m}$ long straight sections. Two diametrically opposite insertions in Points 1 and 5 will house the high-luminosity multipurpose experiments ATLAS and CMS, for which considerable civil construction is required. The more specialised experiments ALICE and LHCb will be installed in the existing caverns of Points 2 and 8 . These insertions will also be equipped with beam injection systems for the two rings. The remaining four insertions will house the other major collider systems: Points 3 and 7 are dedicated to beam collimation, while Points 4 and 6 serve respectively for the RF and beam extraction.

Following a decade of vigorous $R \& D$ and technical validation of the major collider systems in CERN and collaborating laboratories, the LHC construction is now fully underway. Major industrial contracts for the procurement of the superconducting magnets, cryogenic refrigeration plants and other machine systems have been awarded, and their execution successfully started. Some of the collider systems, like the injector complex and the superconducting RF cavities are nearing completion. In parallel with final technical validation and equipment procurement, the upgrading of the existing CERN accelerators and of the LHC tunnel infrastructure have advanced well. The plan for the collider installation has been recently reviewed and agreed with the experimental collaborations, and calls for the start of installation of the LHC arcs for Autumn 2003. Under this plan, the commissioning of the collider will start in spring 2004 with the Octant 7-8 test, and the rings closed by the end of 2005. The first collisions are foreseen for the beginning of 2006, and a seven-month physics run starting in late summer 2006.

\section{LATTICE MAGNETS AND CRYOGENICS}

The LHC lattice design has matured over the past years, both in terms of performance, robustness and flexibility, and validation of critical technologies and engineering solutions [2]. It is composed of 46 half-cells per arc; each half-cell is $53.45 \mathrm{~m}$ long and consists of three twinaperture dipoles, with a magnetic length of $14.3 \mathrm{~m}$, and a twin-aperture quadrupole, $3.1 \mathrm{~m}$ in length. Each dipole is housed in a cold mass together with multipole spool pieces (sextupole, and combined octupole and decapole in every other dipole). Similarly, the quadrupole is assembled in a "short straight section", which also contains orbit correction dipoles and lattice correctors (chromaticity sextupoles, and trim or skew quadrupoles, or Landau damping octupoles, according to its position). For reasons of magnet protection the dipoles and quadrupoles are powered independently, as are the focusing and defocusing quadrupole apertures to allow linear decoupling with a tune split of up to ten units. In total, 1232 main dipoles and 392 lattice quadrupoles will be installed in the collider.

The main dipoles are the most challenging elements of the LHC. They must operate reliably at the nominal field of $8.3 \mathrm{~T}$, reach the ultimate field of $9 \mathrm{~T}$, and show performance stability with thermal cycles and quenching. In addition, they must have good field quality at injection $(0.58 \mathrm{~T})$. For economical production, the low sensitivity to component tolerances and an inherently large tunability range of the coil cross-section are essential features of the design. In order to achieve these goals, CERN has launched since 1995 an intensive R\&D short model programme in house, and a full-length prototype programme in collaboration with industry. A comprehensive summary of the results of these programmes is given in ref. [3].

In 1999, CERN launched a contract for the construction of 90 pre-series dipole cold masses with three firms previously involved in the prototype work, for 30 dipoles each. The first pre-series dipole, shown in Fig. 1, which is suitable for installation in the LHC, was tested in CERN in March 2001. The magnet showed excellent results, with only one training quench at $8.3 \mathrm{~T}$ before surpassing the ul- 
timate field of $9 \mathrm{~T}$ without quench. Further 16 dipoles from all three firms are expected to be delivered by the end of 2001. A call for tender for the fabrication of the full series of the remaining dipole cold masses was recently launched, and the contracts are expected to be adjudicated by the end of 2001. A detailed account of the pre-series programme and plans for the series production of the main dipoles are given in refs. [4] and [5].

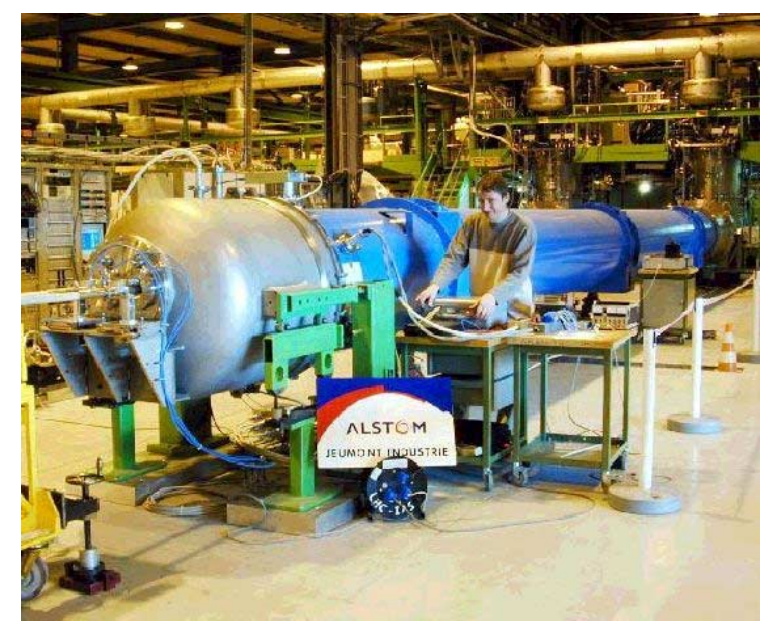

Figure 1: The first pre-series LHC dipole on the test bench in CERN.

The lattice quadrupoles have been designed and prototyped in collaboration between CERN and CEA Saclay (France). To date, three prototypes of the final design have been completed and tested in CERN and Saclay with very good results, confirming the soundness and robustness of the design [6]. The contract for the manufacture of the series quadrupoles has been placed with ACCEL (Germany), and the first pre-series magnets are expected by the end of 2001 [7].

The lattice magnets operate in a static bath of pressurised superfluid helium at a temperature of $1.9 \mathrm{~K}$, cooled by heat exchange with saturated superfluid helium at a pressure of 16 mbar, which flows in a linear heat exchanger tube extending over the $107 \mathrm{~m}$ of each cell [8]. In order to limit the thermodynamic cost of refrigeration, most of the heat loads are intercepted at higher temperatures. The static heat loads are minimised by careful design of the cold mass support posts and the cryostat multilayer reflective shields, designed and validated for large-scale industrial production. The dynamic loads due to the circulating beams are intercepted by the beam screen mounted inside the cold bore and cooled by circulation of supercritical helium between $5-20 \mathrm{~K}$. The LHC cryogenic system therefore uses helium in several thermodynamic states, and is distributed to the magnets by the cryogenic distribution line, which runs parallel to the magnet string and is connected to it every cell.

The LHC will be cooled by eight cryoplants, each with an equivalent capacity of $18 \mathrm{~kW}$ at $4.5 \mathrm{~K}$, located in five points around the collider where all refrigeration, compres-

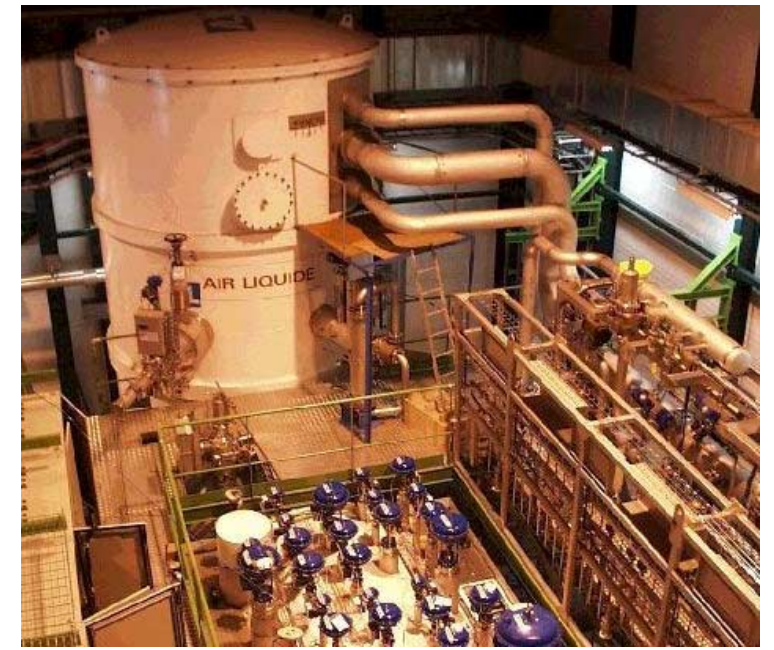

Figure 2: Coldbox of the new $18 \mathrm{~kW}$ refrigerator $(4.5 \mathrm{~K})$.

sion and distribution equipment will be housed at the surface and underground levels. Four of these are the existing refrigerators, upgraded from $12 \mathrm{~kW}$ and used during the final years of LEP operation. Four new refrigerators were specified by CERN in 1997, and contracts adjudicated to Air Liquide (France) and Linde Kryotechnik (Switzerland) for the supply of two plants each. The first of these, shown in Fig. 2, is presently being installed in Point 1.

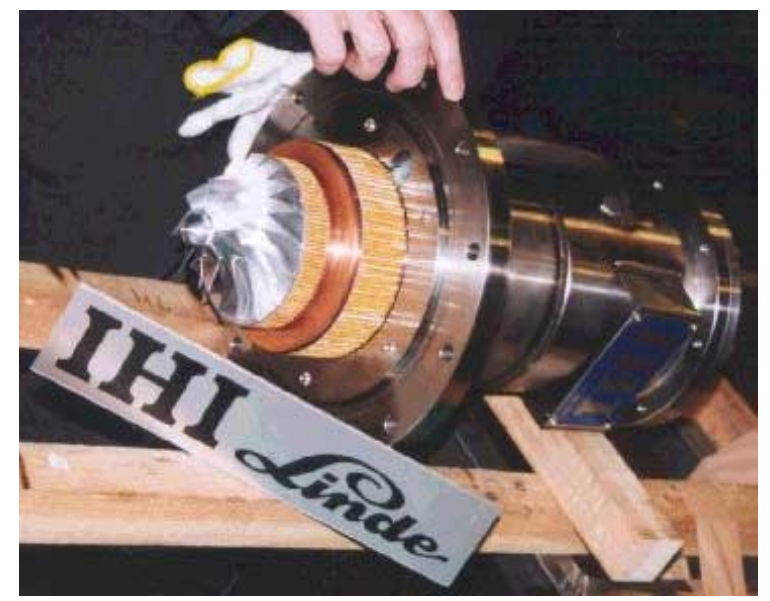

Figure 3: The impeller of the first pre-series cold compressor.

The other major components of the cryogenic system are the eight refrigeration units delivering a total of $19.2 \mathrm{~kW}$ at $1.8 \mathrm{~K}$, based on cold centrifugal compressors. The critical design issues of the cold compressors such as impeller and diffuser design, and drive and bearing technology, have been thoroughly tested on prototypes made in collaboration with industry [9]. In 1998, CERN adjudicated contracts for the supply of four units to a consortium of IHI (Japan) and Linde Kryotechnik (Germany), and another four from Air Liquide (France). The first pre-series unit, shown in Fig. 3, was recently delivered to CERN for testing. 
The qualification tests of the three $120 \mathrm{~m}$ long prototypes of the cryogenic distribution line [10], ordered from industrial partners in 1998, have been recently completed, and the call for tender for the supply of the eight sectors issued. It is expected that the contracts will be adjudicated by the end of 2001.

\section{INSERTIONS}

The optics and layout of the LHC insertions have been optimised with respect to their main function and technical and infrastructure constraints specific to each insertion region. Although the general layout of the LHC has not been modified since 1995, the layout of the insertions has been regularly updated. As an example, the CMS highluminosity insertion has been modified to provide the necessary tuning flexibility for the TOTEM small-angle experiment, and similar features implemented in the ATLAS insertion. The collimation systems in Points 3 and 7, designed to limit the beam losses in the superconducting magnets and background in the experiments, have been considerably reworked to increase their efficiency. Recently, the superconducting RF cavities in Point 4 have been displaced from the tunnel to the experimental cavern in order to solve a major interference with the cryogenic distribution line. These modifications were necessary to resolve the functional or installation bottlenecks that were identified with the advance of the equipment design and its integration in the tunnel.

In spite of functional differences, all insertions contain, moving from the arc towards the middle of an insertion, a dispersion suppressor followed by a matching section, a section of separation dipoles, and in the experimental insertions, the final focus system (low- $\beta$ triplet). The warm areas house the forward experimental detectors, beam injection and extraction systems, collimators and the RF system and beam instrumentation. A significant part of the magnet and other collider systems installed in the insertions is supplied by accelerator laboratories world-wide as part of the contributions of respective non-member countries to the LHC. In view of the complicated interfaces, advanced stage of equipment design and tight budgets, frequent contacts and close collaboration between laboratories have been established. Configuration control and monitoring of progress have become simpler with the advent of the engineering document management system (EDMS) [11], allowing unified access to the approved and up-to date technical documents world-wide.

The low- $\beta$ triplet is located at $23 \mathrm{~m}$ on each side of the interaction point, and is composed of four single aperture quadrupoles with a coil aperture of $70 \mathrm{~mm}$. It is cooled with superfluid helium at $1.9 \mathrm{~K}$ using an external heat exchanger system capable of extracting up to $10 \mathrm{~W} / \mathrm{m}$ of power deposited in the coils by the secondaries emanating from the p-p collisions. Two types of quadrupoles are used in the triplet, $6.6 \mathrm{~m}$ long MQXA magnets designed and developed by KEK (Japan), located on either side of the two central
$5.7 \mathrm{~m}$ long MQXB magnets designed and built as a single cold mass by FNAL (USA). The magnets are powered in series with $7 \mathrm{kA}$, with an additional inner loop of $5 \mathrm{kA}$ for the MQXB magnets. Together with the dipole orbit correctors and multipole spool pieces supplied by CERN, the three low- $\beta$ quadrupoles are completed and cryostated by FNAL. The cryogenic feedboxes, providing a link to the cryogenic distribution line and power converters, are designed and built by LBNL (USA).

Alongside the LHC main dipoles, the high-gradient, wide-aperture low- $\beta$ quadrupoles are the most demanding magnets in the collider. They must operate reliably at $215 \mathrm{~T} / \mathrm{m}$, sustain extremely high heat loads in the coils and radiation dose during their lifetime, and have a very good field quality within the $63 \mathrm{~mm}$ aperture of the cold bore. In order to validate their design choices, KEK and Fermilab have launched in 1996 comprehensive R\&D programmes comprising a number of short model magnets, refs. [12] and [13]. Both programmes, completed last year, were successful in fulfilling the design goals and demonstrating the LHC operational requirements, following which full-length prototype magnets were constructed.

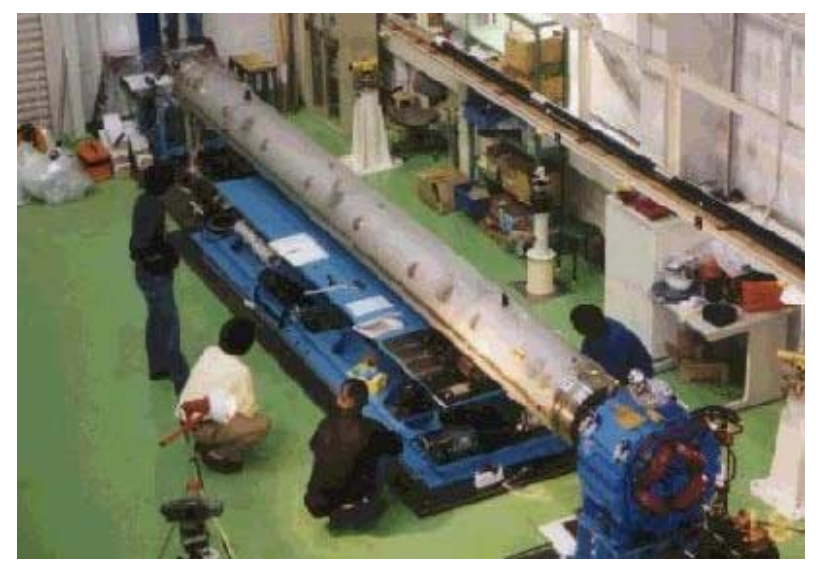

Figure 4: Prototype MQXA low- $\beta$ quadrupole on the warm test bench in KEK.

The $6.6 \mathrm{~m}$ long prototype MQXA quadrupole is shown in Fig. 4 during reception in KEK. The magnet was tested in KEK in the newly built $1.9 \mathrm{~K}$ vertical test facility in April 2001, and surpassed the ultimate gradient in LHC of $215 \mathrm{~T} / \mathrm{m}$ in two training quenches. The training was confirmed after a thermal cycle, when the magnet reached $230 \mathrm{~T} / \mathrm{m}$ in two quenches. In Fig. 5, the $5.7 \mathrm{~m}$ long MQXB prototype is shown ready for cryostating. This magnet was tested in the upgraded $1.9 \mathrm{~K}$ horizontal test station in FNAL in May 2001, and reached the target for quench training of $230 \mathrm{~T} / \mathrm{m}$ in eight quenches. On the basis of these excellent results, the series fabrication of the low- $\beta$ quadrupoles has been launched. In total, $16 \mathrm{MQXA}$ quadrupoles will be supplied by KEK, built in Japanese industry, and 16 MQXB quadrupoles will be built in Fermilab. The first triplet is expected in CERN by the end of 2002.

An important result of both $R \& D$ programmes, con- 


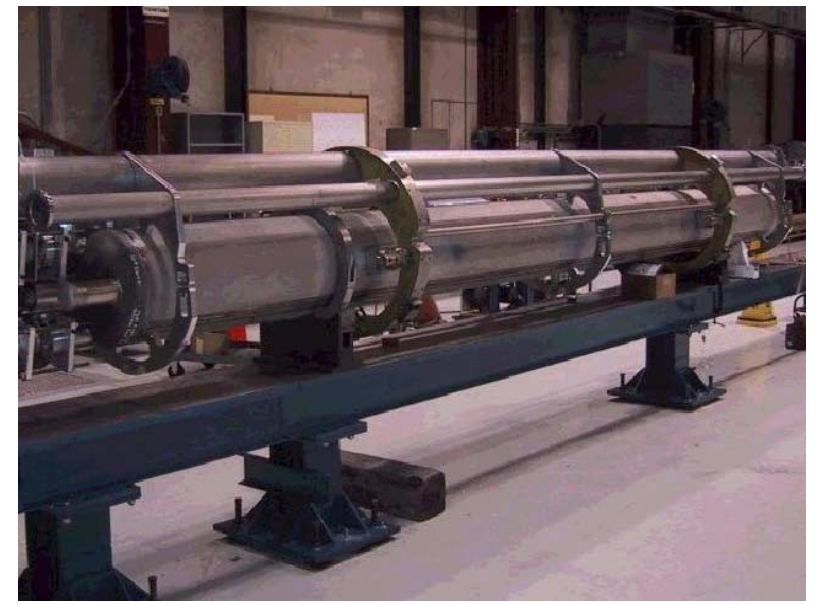

Figure 5: Prototype MQXB low- $\beta$ quadrupole ready for cryostating in Fermilab.

firmed by the quadrupole prototypes, is that the field quality is considerably better than initially expected. As a result, the number and strength of the multipole correctors could be considerably reduced and their arrangement simplified. In view of the fact that the low- $\beta$ quadrupoles govern the dynamic aperture and luminosity of the LHC at top energy, these results give confidence in achieving the design performance of the collider.

The separation dipoles in the experimental insertions bring the two beams onto a colliding orbit from their nominal separation of $194 \mathrm{~mm}$ in the arcs. To limit the beambeam effects, the first separation dipole D1 is immediately upstream of the low- $\beta$ triplet. In the high-luminosity insertions, the radiation levels are high and more robust warm magnets (MBXW) are used. In the ALICE and LHCb insertions, D1 is a stronger superconducting magnet (MBX) allowing more space for the injection systems. In all cases, the D2 separation dipole (MBR) is a twin aperture superconducting magnet.

The MBX and MBR dipoles are designed and built by BNL (USA) on the basis of the RHIC lattice dipole, and feature a $10 \mathrm{~m}$ long, $80 \mathrm{~mm}$ aperture coil. Magnets with identical coils and similar yoke configurations are also used for the D3 and D4 separation dipoles in the RF insertion, where the beam separation is increased to $420 \mathrm{~mm}$ to accommodate the superconducting cavities. All magnets operate at a field of up to $3.8 \mathrm{~T}$ at $4.5 \mathrm{~K}$. BNL has built and tested $3 \mathrm{~m}$ prototypes of the twin aperture $\mathrm{D} 4$ magnet, which showed very satisfactory quench performance and field quality [14]. The series production of these magnets has started in BNL, see Fig. 6, and the first units will be delivered to CERN by the end of 2001.

A number of warm magnets will be installed in the beam cleaning and high-luminosity experimental insertions. In total, 45 warm dipoles and 48 quadrupoles are required. The most advanced are the MQW twin aperture quadrupoles, featuring a pole aperture of $46 \mathrm{~mm}$, which are designed and produced in collaboration with TRIUMF

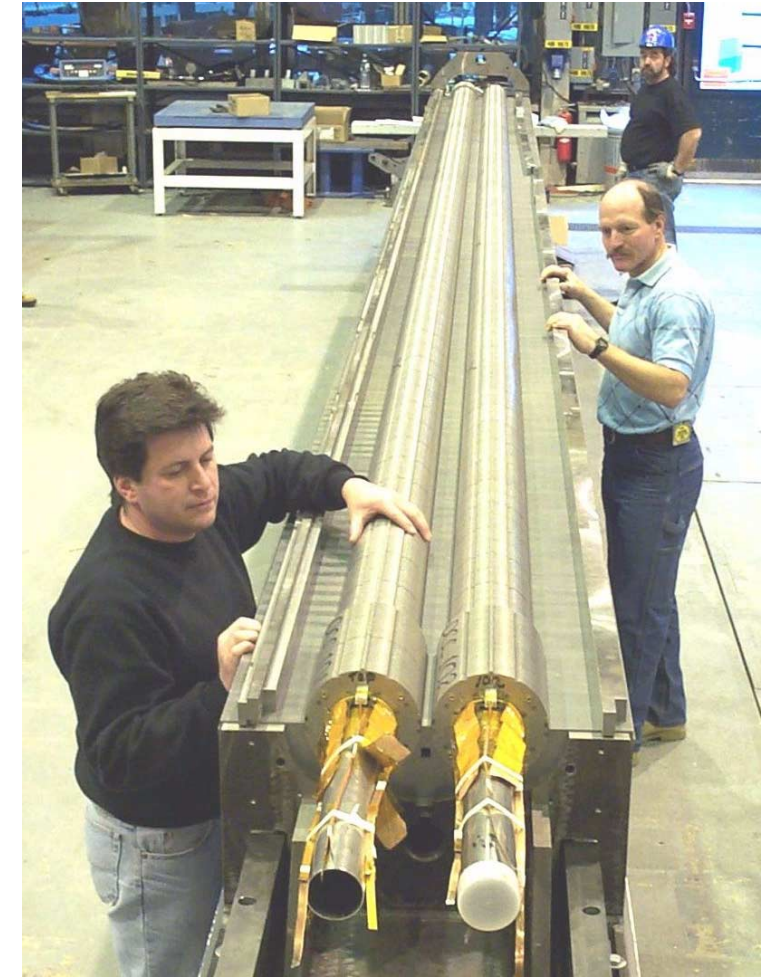

Figure 6: Assembly of the first series D2 separation dipole in BNL.

(Canada) [15]. After a prototype in 1998, a pre-series MQW quadrupole has been built by Alstom (Canada) and tested in CERN in March 2001; the first series magnets are expected by the end of 2001. The warm MBW dipoles, used in the D3-D4 dogleg in Points 3 and 7, as well as the MBXW dipoles in the ATLAS and CMS insertions, are designed and built in collaboration with BINP (Russia), which is also providing warm orbit corrector dipoles and compensator magnets for the ALICE and LHCb experimental dipoles.

The optics flexibility of the LHC insertions is provided by the individually powered quadrupoles in the dispersion suppressors and matching sections. CERN has developed two quadrupoles for this purpose, one featuring a two-layer $56 \mathrm{~mm}$ aperture coil (MQM) [16], and the other an enlarged, $70 \mathrm{~mm}$ aperture four-layer coil (MQY) [17]. Both quadrupoles use an $8 \mathrm{~mm}$ wide cable, so that the nominal current is less than $6 \mathrm{kA}$, substantially simplifying the warm and cold powering circuits. Following the successful model programme, contracts for the supply of 94 MQM and 20 MQY quadrupoles have been adjudicated in 2000 , and first pre-series magnets are expected beginning of 2002 .

\section{CIVIL CONSTRUCTION AND INSTALLATION}

Although the existing infrastructure of the LEP tunnel and surface buildings is used to the utmost for housing the 
LHC, a considerable amount of new civil construction is needed for the ATLAS and CMS experiments, for the two transfer tunnels from the SPS, and for the technical buildings and assembly halls. The ground breaking on all locations started in 1998 and in spite of a number of unforeseen problems related mainly to the tunnel geology, the underground and surface works have advanced well. Following the decision to stop LEP in December 2000, the pace of the construction work has increased. In May 2001, an important milestone was reached with the junction of the TI2 transfer tunnel to the main LHC tunnel. The major works, the transfer tunnels and experimental caverns are now on schedule to be delivered to the LHC machine and experiments by mid 2003.

The tight constraints of the LHC tunnel, the large amount of equipment that needs to be transported and installed (over 40000 tons), and the limited time for installation require detailed preparations, including CAD and full-scale simulations. On the basis of verification of the tunnel geometry performed in 1999, 3D digital mock-ups have been developed for all tunnel sections. These studies have allowed to identify previously unknown areas of interference between major equipment, and to validate transport and installation scenarios. As part of these preparations, the recent experience gained in the assembly of the first half of the Test String 2 [18], featuring a full $107 \mathrm{~m}$ long cell comprising prototype dipoles and quadrupoles, has been of great value in validating the techniques and tools developed for installation and interconnections of the lattice magnets. Similar activities are planned in the future to optimise the procedures and train the installation teams.

\section{OUTLOOK}

Following a decade of comprehensive R\&D and technical validation of the major collider systems, the LHC construction is now fully underway. Major industrial contracts have been awarded and are in execution for the procurement of the magnet, cryogenics and other systems. Initial experience shows that industry is in general well prepared, but that the quality control procedures required by CERN must be strictly implemented for production of all components and assemblies. Excellent and constructive relations have been established with accelerator laboratories worldwide, and the fabrication of equipment supplied as part of the contributions of non-member countries is advancing well. Civil construction and upgrading of the technical infrastructure is proceeding as planned, giving confidence in meeting the tight schedule for collider installation and first circulating beams, agreed with the experiments for the beginning of 2006 .

\section{ACKNOWLEDGMENTS}

The work reported in this paper is the result of the technical expertise, ingenuity and dedication of a large number of teams and groups in CERN, in the collaborating labo- ratories world-wide and in industry. The author wishes to acknowledge their excellent results, and is grateful for the contributions received.

\section{REFERENCES}

[1] The LHC Design, Beam performance, and Machine Parameters \& Layouts, http://lhc.web.cern.ch/lhc.

[2] L. R. Evans, "LHC Accelerator Physics and Technology Challenges”, Proc. PAC'99, New York, March 1999, pp. 2125.

[3] C. Wyss, "The LHC Magnet Programme: From Accelerator Physics Requirements to Production in Industry", Proc. EPAC'2000, Vienna, Austria, June 2000, pp. 207-211.

[4] C. Wyss, "The LHC Main Magnets: A Status Report", presented at this Conference.

[5] M. Bajko et al., "Final Prototypes, First Pre-Series Units and Steps Towards Series Production of the LHC Main Dipoles", presented at this Conference.

[6] T. Tortschanoff et al., "Performance of the Series-Design Prototype Main Quadrupoles for the LHC", Proc. EPAC'2000, Vienna, Austria, June 2000, pp. 2172-2174.

[7] J. Billan et al., "Performance of the Prototypes and Start-up of Series Fabrication of the LHC Arc Quadrupoles", presented at this Conference.

[8] Ph. Lebrun, "Cryogenics for the Large Hadron Collider", IEEE Trans. Appl. Superconductivity, Vol. 10 (2000), No. 1, pp. 1500-1506.

[9] L. Tavian, "Large Cryogenic Systems at $1.8 \mathrm{~K}$ ", Proc. EPAC'2000, Vienna, Austria, June 2000, pp. 212-216.

[10] W. Erdt, G. Riddone, R. Trant, "The Cryogenic Distribution Line: Functional Specification and Conceptual Design", Adv. Cryog. Eng. 45A (2000), pp. 1387-1394.

[11] The LHC Technical Coordination \& Planning, http://lhc.web.cern.ch/lhc/tcp/WelcomeTCP.html.

[12] T. Shintomi et al., "Progress of the LHC Low- $\beta$ Quadrupole Magnets at KEK", IEEE Trans. Appl. Superconductivity, Vol. 11 (2001), No. 1, pp. 1562-1565.

[13] N. Andreev et al., "Status of the LHC Inner Triplet Quadrupole Program at Fermilab", IEEE Trans. Appl. Superconductivity, Vol. 11 (2001), No. 1, pp. 1558-1561.

[14] E. Willen et al., "Superconducting Dipole Magnets for the LHC Insertion Regions", Proc. EPAC'2000, Vienna, Austria, June 2000, pp. 2187-2189.

[15] G. S. Clark et al., "A Twin Aperture Resistive Quadrupole for the LHC", IEEE Trans. Appl. Superconductivity, Vol. 10 (2000), No.1, pp. 147-149.

[16] J. Lucas et al., "Performance of the Single and Twinaperture Models of the $6 \mathrm{kA}$ Superconducting Quadrupole for the LHC Insertions", IEEE Trans. Appl. Superconductivity, Vol. 11 (2001), No. 1, pp. 1645-1648.

[17] G. Kirby et al., "Performance of the $1 \mathrm{~m}$ Model of the $70 \mathrm{~mm}$ Bore Twin-Aperture Quadrupole for the LHC Insertions", IEEE Trans. Appl. Superconductivity, Vol. 11, (2001), No. 1, pp. 1641-1644.

[18] F. Bordry et al., "The Commissioning of the LHC Test String 2", presented at this Conference. 